\title{
The Development Research of 5G Communication Technology Promotes the Internet of Things Industry Chain
}

\author{
Chao Duan \\ Guangzhou College of Technology and Business, Guangzhou, China
}

Keywords: 5G, Communication technology, Internet of things, Industry chain

\begin{abstract}
The current Internet of Things development is relatively fast, and 5G communication technology has a relatively good prospect under Internet of Things situation, which has a great impact on Internet of Things industry chain development. In order to do a continuous development of 5G communication technology, reasonable measures must be taken. It is to enhance the industrial chain mode of the Internet of Things development form. This article discusses the concept and development status of 5G communication technology from the practical point of view, and then discusses how to promote the development of the Internet of Things industry chain.
\end{abstract}

\section{Introduction}

Today, with the continuous improvement of the material and cultural life of the masses, mobile networks and the Internet of Things(IoT) have been integrated into all aspects of real life, and people's requirements for network communication and the IoT have become higher and higher. Therefore, to meet the diversity of people and meet the needs of life, we must vigorously develop 5G communication technology and apply it to the IoT industrial chain, so as to further improve the service quality and operating efficiency of the IoT, and realize the transformation of the entire IoT industrial chain as soon as possible. Research on the development of the IoT industry chain from the perspective of $5 \mathrm{G}$ communication technology is also very practical [1].

\section{Basic Characteristics of 5G Communication Technology}

$5 \mathrm{G}$ technology is a communication technology extended on the basis of 2G, 3G, 4G communication technology (as shown in Figure 1), its essence is network convergence, through effective data connection for people and things, things and things, people and people provide a safe and reliable information transmission channel [1]. The basic characteristics of 5G communication technology are mainly reflected in below aspects:

First, the coverage of $5 \mathrm{G}$ communication technology is very wide. Compared with $4 \mathrm{G}$, the $5 \mathrm{G}$ communication technology almost achieves uninterrupted full coverage, so as to ensure that users are provided with mobile stronger, higher continuity, and smoother data transmission [2].

Second, 5G communication technology has higher capacity. In local hotspots, customers can experience ultra-high data transmission rates to satisfy users [1]. The demand for high traffic density of the network brings users a more convenient and comfortable operating experience.

Third, 5G communication technology has low connection energy consumption. In the current era of advocating energy-saving, environmental protection and green development, 5G communication technology has a large amount of data [3]. At the meantime, it has very low energy consumption and is suitable for new intelligent fields such as smart cities and smart agriculture. It can reduce energy consumption and costs while meeting high index requirements.

Fourth, 5G communication technology has very low latency, higher reliability, the information only needs a few milliseconds delay from the output to the terminal, and the information has a very high reliability in the transmission process, which can meet the requirements for low information transmission in the field of automobile driverless and industrial control [2]. 


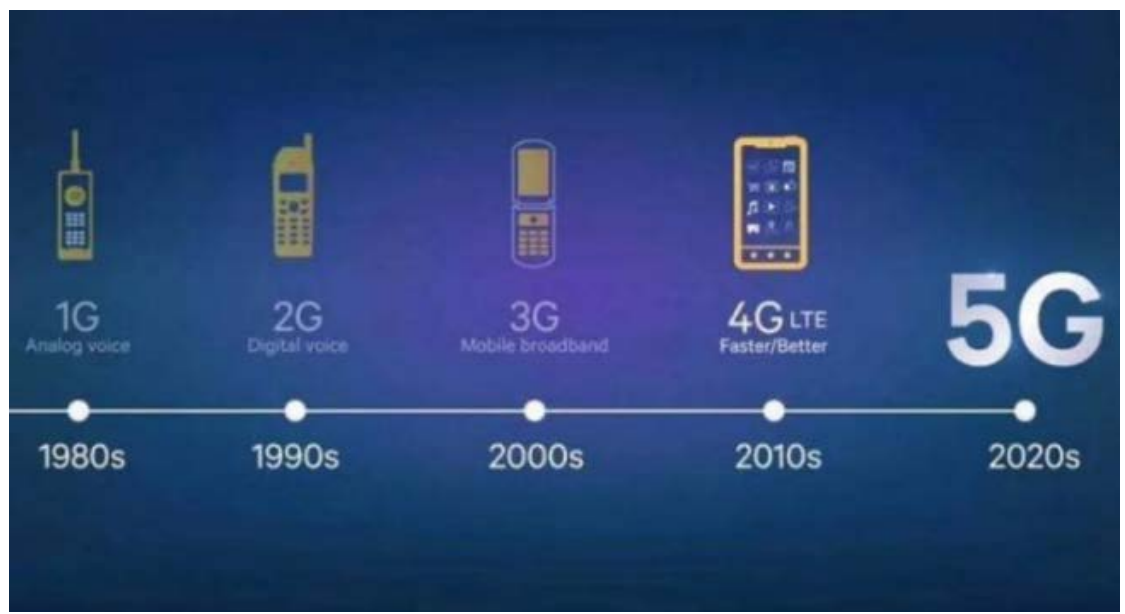

Fig.1 5G Development Trajectory

\section{The Influence of 5G Technology on the Development of Iot Industry Chain}

\subsection{Mutual Development}

With the proposal of 5G communication technology, the IoT industry has entered a new platform. Through 5G communication, rapid terminal industry service construction can be achieved, the speed of the IoT industry is accelerated, and industrial communication has achieved online and offline synchronous mirror communication [3]. The IoT resources have more industrial advantages and are more in line with the overall development needs and construction standards.

\subsection{Security and Privacy Impact}

Privacy and security are important IoT components of industry chain. After the application of 5G communication technology, the interoperability of the IoT will be further developed, and the security and privacy will also be greatly improved. At the current stage, the transparency of the IoT industry chain is constantly increasing. If security and privacy cannot be improved, it will cause the IoT industry chain to fall into a predicament, causing serious impact and huge losses. Today is the era of the IoT [3]. People can gradually change their past lifestyles, use artificial intelligence systems to update their lifestyles, improve their quality of life, and put more energy into work and research and development.

\subsection{Development Cost Impact}

With the continuous research and development process of 5G communication technology, some technologies have begun to test, the purpose is to collect more data and information, improve 5G communication technology, so that it can be maximized, and can be reasonably applied. In IoT industry chain development, the impact of 5G communication technology is huge, so in terms of cost, it is necessary to adopt reasonable methods to deal with it, and then achieving the desired effect, improving the problem, and making it develop rapidly [4]. In the early stage of the development of the IoT, reading and writing equipment and electronic tags are expensive, resulting in the phenomenon of high cost of the IoT development, to a certain extent, inhibiting the large-scale development of the IoT [3]. Therefore, in the development and application of 5G communication technology, it is necessary to strictly control the cost and pay close attention to the cost of the IoT industry chain.

\section{The Significance of 5G Mobile Communication Technology Applied to the Internet of Things Industry Chain}

\subsection{Improve the Iot Industry Chain}

Judging from China's communication technology development status, the current communication 
standards and communication technologies in various industries are gradually being optimized and improved. Against this background, if the IoT industry chain wants to fully integrate with the development trend of the times and extend the industry chain, it must rely on high-tech communication technologies to support the development of the IoT industry [4]. This is mainly because $5 \mathrm{G}$ mobile communication technology can not only be applied to real communication scenarios and actual environments in all walks of life, but also developed efficiently at the level of information services. Therefore, the full 5G communication technology application in the IoT industry chain can give full play to the objective advantages of $5 \mathrm{G}$ communication technology, thereby promoting the development and progress of the IoT industry. In addition, the completion of interconnection and communication between networks according to current unified standards is more conducive to the establishment of a scientific and highly complete industrial chain of the IoT, in order to improve the industrial value of the IoT.

\subsection{G Technology Expands Application Technologies for the Development of the Internet of Things}

In the era of traditional technology, the IoT industry can only be limited to its own industries and fields, and it is difficult to diversify. When 5G was born in the world, it provides more possibilities for the IoT industry development [5]. With the great supporting of 5G technology, the IoT industry can develop and progress. Moreover, its industrial coverage has also diversified. 5G mobile communication technology the city-level high-tech communication technology is one of the integrated high-tech technologies. Among them, the technologies that can be applied to the IoT industry chain to exert their advantages include NOMA technology and dense networking and MIMO basic methods [5].

\subsubsection{Noma Technology}

The main function of this technology is: it can significantly reduce the interference of various external factors on information transmission, and it has a good role in ensuring that the system is not interfered by external factors and enhances the effect of communication transmission. Applying this technology to the development of the IoT industry chain can extend the value of the IoT industry.

\subsubsection{Dense Networking Technology}

This technology is mainly based on the establishment of network verification slices, which mainly includes two core technologies, namely SDN and NFV. If professional developers can properly use this technology in the IoT industry and industry, they can achieve accurate information transmission and strict control of information. In addition, the dense networking technology can intelligently identify and sense various network data, and increasing data transmission speed on the network to ensure the transmission quality and transmission data transmission terminal speed.

\section{The Application Path of 5G Communication Technology in Iot Development}

\subsection{Adjust the Deployment of Local Industry Chain}

Although the 5G mobile communication technology development which will provide important technical support for the development of the IoT industry chain, for the effective integration of 5G communication technology and the IoT industry chain, the deployment of the local industry chain still needs to be adjusted reasonably. On the one hand, since 5G mobile communication technology has not yet been fully commercialized, when deploying the IoT industry chain, it is necessary to fully consider the industrial advantages and disadvantages of various regions, and make the advantages of various regions for industrial module layout to ensure the stability of the IoT industry chain and create good basic conditions for the application of 5G mobile communication technology [6]. On the other hand, due to the great changes in the consumer groups of the IoT, the layout of the industrial chain of the IoT also needs to carry out in-depth data investigation and analysis in response to consumer needs, and determine a reasonable industrial chain layout based on the results of big data analysis Strategy. 


\subsection{Commercial Development for the Internet of Things}

In the future of technological process, the IoT industry chain fully relies on $5 \mathrm{G}$ communication technology to achieve the ultimate goal of fully integrating communication methods and communication measures, and complete the transformation and diversified development of the IoT industry. 5G technology brings users a more real feeling of mobile communication, makes users immersive, and truly achieves the effect of interconnection between people and objects [6].

\subsection{Improve the Overall Growth Rate of the Internet of Things Industry}

With the help of 5G communication technology, the IoT industry chain can achieve a faster growth rate, and then continue to promote the IoT industry chain development, seeking higher economic benefits. The results of relevant research data show that in the past three years, the IoT industry has reached a CAGR of 35\%. In 2016, the IoT industry achieved a total market value of one trillion yuan. The application of 5G communication technology in communication industry not only realizes the immediacy and authenticity of communication transmission, but also meets the development needs of the IoT industry chain, and ensures the accuracy and effectiveness of the IoT industry chain communication. The development of China has provided a steady flow of technical power [7].

\subsection{G Communication Technology Promotes the Commercial Development of the Internet of Things Industry}

5G communication technology can effectively promote the commercial development of the IoT industry [6]. The development of 5G communication technology accelerates the integration of the IoT and the mobile Internet. It changes the traditional communication model and brings users a more convenient and efficient 5G experience. It affects people's daily life and work status. 5G communication technology effectively connects people to people, people to things, things to things, expands the business scope of the IoT, accelerates the integration of various industries, and promotes the optimization and upgrading of traditional industries and emerging industries [8]. The rise of the Internet has driven the development of society and economy, which has brought great business value to the IoT. In the process of continuous development and improvement of 5G communication technology, the IoT industry will gradually develop towards commercialization [7]. The popularization of IoT applications has made more and more mobile terminal devices connected to 5G networks, showing the IoT the immense commercial value has accelerated the commercialization process of the IoT industry and promoted the further social economy development.

\subsection{G Communication Technology Expands the Influence Range of the Internet of Things}

The 5G communication technology integrates the IoT and the mobile communication network, making the IoT have the characteristics of a mobile communication network. The coverage of the mobile communication network is as large as the coverage of the IoT. The flow rate of the mobile communication network is as fast as possible, the traditional rate of information of the IoT will be as fast. 5G communication technology overcomes the problems of narrow coverage of wired networks and slow data transmission rate of $4 \mathrm{G}$ communication technology, provides a suitable network environment for the IoT, so that the scale of the IoT has been greatly expanded. The impact of networking will cover the world, connect the world, and realize economic globalization. As the 5G communication technology continues to mature, the global IoT market is also expanding [8]. According to relevant surveys, the development of 5G communication technology is still in its infancy in 2015. At this time, the global IoT market is only 4,000 One billion yuan, and related predictions that by 2020, after further promotion of 5G communication technology worldwide, the global IoT market will reach three trillion yuan, an increase of more than 7 times from 2015, and the number of IoT connections will also In these five years it has tripled [8].

\section{Summary}

In summary, 5G communication technology has brought tremendous supporting the IoT industry chain development in terms of hardware industry, unified communication standards, and application 
of advanced technologies. It also has very good application prospects in medical care fields, construction, and smart furniture. In order to achieve long-term stable the entire industrial chain development, it is also necessary to pay more attention on the problems of local industrial chain deployment, pre-cost control, and information security protection.

\section{Acknowledgements}

This paper is supported by Guangdong Youth Fund Project, No. 2019KQNCX232.

\section{References}

[1] Zhao, G.F., Chen, J., Han, Y.B. Overview of Key Technologies in 5G Mobile Communication Networks. Journal of Chongqing University of Posts and Telecommunications, no. 4, pp. 41-45, 2018.

[2] You, X.H., Pan, Z.W., Gao, X.Q. 5G Mobile Communication Development Trends and Several Key Technologies. Information Science, no.5, pp.551-553, 2016.

[3] Wang, Y.T. 5G Communication Technology Promotes the Analysis of the Development of the Internet of Things Industry Chain. Information and Communication, no. 5, pp. 22-25, 2018

[4] Liu, L.T., Liu, C.Y., Zhang, C.W. 5G Communication Technology Promotes the Development of the Internet of Things Industry Chain. Digital Communication World, no. 12, pp. 53-55, 2018.

[5] Gao, F.B., Zhang, X.J. 5G Wireless Communication Technology and its Impact on the Development of the Internet of Things Industry Chain. Digital Communication World, no. 10, pp. 74-75, 2018.

[6] Lu, F.F. Research on the Development of the Internet of Things Industry Chain from the Perspective of 5G Communication Technology Development. China's Strategic Emerging Industries, no. 4, pp. 112-114, 2018

[7] Zhang,W. Analysis of 5G Communication Technology to Promote the Development of the Internet of Things Industry Chain. Information and Communication, no. 9, pp. 221-224, 2017.

[8] Jiang, Z.F. 5G Promotes the Development of Internet of Things Technology and Applications. High Technology and Industrialization, no.10, pp.38-41, 2017. 